\title{
Serum Ischemia-Modified Albumin, Fibrinogen, High Sensitivity C- Reactive Proteins in Type-2 Diabetes Mellitus without Hypertension and Diabetes Mellitus with Hypertension: A Case-Control Study
}

\author{
Sushith Sushith ${ }^{1}$, Herijenahalli Nagaraju Krishnamurthy ${ }^{1}$, Shridhar Reshma ${ }^{1}$, \\ D'Sa Janice ${ }^{1}$, Gopal Madan ${ }^{1}$, Kumar Jeppu Ashok ${ }^{1,2}$, \\ Mangalore Balakrishna Prathima*1, Bhuvanesh Sukhlal Kalal*3,4
}

\begin{abstract}
Background: The objective of this study was to determine the levels of serum ischemia-modified albumin (IMA), fibrinogen (FIB) and high sensitivity C-reactive protein (hs-CRP) in type 2 diabetes mellitus (T2DM) patients with hypertension (HT) (DMT2HTN) and without HT (DMT2). Also, their association with certain biochemical and physical factors were studied to identify possible risk factors that lead to cardiovascular complications.

Methods: Fasting blood samples were collected from 35 DMT2 or DMT2HTN patients each to analyze differences in serum and plasma levels of IMA, hs-CRP, FIB, total cholesterol (TC), high and low density lipoproteins (HDL and LDL), triglyceride (TG), hemoglobin A1c (HbA1C), glycated hemoglobin and creatinine.

Results: In DMT2 and DMT2HTN patients, IMA, hs-CRP, FIB, TC, TG, HDL, LDL, glycated hemoglobin and creatinine levels, including body mass index (BMI) and waist-to-hip ratio (WHR), were significantly higher relative to healthy controls. In addition, the levels of IMA, hs-CRP and FIB levels showed a strong link to BMI, WHR, TC, TG, LDL and glycated hemoglobin. Lastly, both DMT2 and DMT2HTN patients demonstrated a significant reduction in HDL.

Conclusions: DMT2 and DMT2HTN patients have a greater risk of developing cardiovascular related complications. This study suggests that quantifying hs-CRP, IMA and FIB levels can help diagnose the risk of developing complications during the early stages of metabolic and cardiovascular disease. Overall, the specific risk factors may be used for early identification of cardiovascular complications to decrease mortality and morbidity in T2DM patients.
\end{abstract}

Keywords: Diabetes Mellitus, Fibrinogen, Hemoglobin, High sensitivity $\mathrm{C}$ reactive proteins, Hypertension, Ischemia modified albumin.

\section{Introduction}

Diabetes is endemic worldwide and the rise in cases puts a significant economic burden on

countries in Asia, Latin America, and Africa (1). It is projected that by the year 2030, India will

1: Department of Biochemistry, A. J. Institute of Medical Sciences and Research Centre, Mangaluru 575004, Karnataka, India.

2: International Medical School, Management and Science University, Shah Alam, Selangor 40100, Malaysia.

3: A. J. Research Centre, A. J. Institute of Medical Sciences and Research Centre, Mangaluru 575004, Karnataka, India.

4: Ophthalmology, Visual \& Anatomical Sciences, Wayne State University, Kresge Eye Institute, Detroit, Michigan 48201, United States of America.

*Corresponding authors: Mangalore Balakrishna Prathima; Tel: +919739309942; E-mail: mbprathima@gmail.com

\& Bhuvanesh Sukhlal Kalal; Tel: +919739309942; E-mail: bhuvanesh611@gmail.com.

Received: 26 Apr, 2020; Accepted: 10 May, 2020 
have about 98 million people with type 2 diabetes mellitus (T2DM) (2). Diabetes is associated with a number of complications including diabetic retinopathy, nephropathy, neuropathy and cardiovascular problems in multiple organs, which, together, can increase the mortality and morbidity in these patients (3). The risk of complications is commonly assessed by measuring ischemia-modified albumin (IMA), hemoglobin A1c (HbA1c) and glycated hemoglobin levels.

Hypertension (HT) is closely related to diabetes, the symptoms of which are initially parallel but may eventually outstrip it (4). Indeed, $40-60 \%$ of diabetes cases have elevated blood pressure (BP). The combined onset of HT and diabetes will lead to the development of stroke and myocardial infarctions.

HT pathophysiology in diabetics involves complex interactions with many environmental factors and between the nervous, cardiovascular and renin-angiotensin systems (5). It also includes physiological changes such as increased levels of blood sugar levels and abnormal levels of lipids, increased oxidative stress, chronic inflammation, hypoxia and ischemia $(6,7)$.

Given the limited data available on the relationship between the metabolic profile of diabetic and hypertensive patients, the study aimed to evaluate serum levels of IMA, fibrinogen (FIB) and high sensitivity C-reactive proteins (hs-CRP) and whether these concentrations correlate with other biological markers in DMT2HTN and DMT2 patients. The direct relation between the patients with DMT2 and DMT2HTN has not been examined to the best of our knowledge. Hence, we conducted a case-control study to evaluate the clinical and laboratory parameters in DMT2 and DMT2HTN patients compared to healthy people.

\section{Materials and methods \\ Study Population}

The case-control study was conducted between 2012 to 2015 in individuals between 35-65 years of age who were diagnosed with type 2 diabetes without HT (newly diagnosed or known diabetic on treatment) or with HT and serum creatinine concentration of $<2 \mathrm{mg} / \mathrm{dl}$.
HT was defined as having a systolic BP (SBP) of approximately $\geq 140 \mathrm{mmHg}$ or diastolic BP (DBP) of approximately $\geq 90 \mathrm{mmHg}$ or a history of HT treatment. The research omitted participants with a known history of febrile illness, renal failure, chronic diseases, benign and malignant disorders. Within the control group, individuals had no known chronic disease and drug use and a normal fasting plasma glucose score. Prior to the start of the study, ethical approval was obtained from the Institution Ethics Committee (Ref no. ECR/348/INST/KA/2013) and informed consent was obtained from all participants.

The study population consisted of 105 individuals divided into the following categories: Group I (n=35): Age and sex-matched normal individuals as controls; Group II $(\mathrm{n}=35)$ : Diagnosed cases of DMT2; Group III ( $\mathrm{n}=35)$ : Diagnosed cases of DMT2HTN.

\section{Biochemical Parameters}

In order to calculate the body mass index (BMI) and waist-to-hip ratio (WHR), physical parameters were measured including height, weight, waist and hip circumference. Using aseptic techniques, after 8-12 hours of fasting, 7 $\mathrm{mL}$ of blood was obtained from the antecubital vein in ethylenediaminetetraacetic acid (EDTA, 2 $\mathrm{mL})$ and plain vacutainers $(5 \mathrm{~mL})$.

For subsequent biochemical analysis, blood samples were centrifuged to separate plasma and serum. Serum was used for analyses of IMA, FIB, hs-CRP, total cholesterol (TC), triglycerides (TG), high-density lipoprotein (HDL), low-density lipoprotein (LDL) and creatinine. Plasma was used exclusively for $\mathrm{HbA1c}$ analysis.

IMA was estimated using Cobalt Chloride kit (Merck, Germany) and DTT (Hi Media, Mumbai, India) at a wavelength of $470 \mathrm{~nm}$ conducted using a spectrophotometer (Manti Lab Solutions, India) (8). Serum FIB and hs-CRP were estimated Commercial kits (Transasia Bio-Medicals, Mumbai, India) using coagulation analyser (COLAB 6000, Chicago USA) and turbidimetric immunoassay semi-autoanalyzer (Mispa i2, Agappe, India). Glycated hemoglobin and $\mathrm{HbA1C}$ were measured using a method called Ion 
Exchange Resin. Cholesterol was measured using enzymes including Cholesterol OxidasePeroxidase 4-aminoantipyrine. Serum TG was also measured using enzymes, specifically, GPOESPAS (Glycerol 3 Phosphate OxidasePeroxidase N-Ethyl-N-Sulfopropyl-n-anisidine). All these kits were from Tulip Diagnostics, Goa, India LDL was calculated using the Friedewald formula:

LDL cholesterol mg/dl $=\mathrm{TC}-[\mathrm{HDL}$ Cholesterol $+\mathrm{TG} / 5]$

Serum creatinine was estimated using a modified Jaffes method (9). HbA1C was measured by Ion Exchange Resin using a commercial kit (Tulip Diagnostics).

\section{Statistical analysis}

Statistical analysis was conducted using version 11.0 of the Social Sciences Statistical System (SPSS Inc, Chicago, IL). Quantitative data was represented as the mean \pm standard deviation
(SD). ANOVA was used for multiple group comparisons. The relationship between variables was calculated using the Karl Pearson's coefficient. A p-value $<0.05$ was deemed statistically significant.

\section{Results}

Table 1 summarizes the demographic, clinical, and laboratory findings in group I, II and III. There was no significant difference in the mean age for all three groups $(\mathrm{p}=0.6)$.

We first compared BMI in DMT2 patients (24.68 \pm 2.515$)$, DMT2HTN patients $(31.043 \pm$ $3.641)$ and controls $(19.238 \pm 3.231)(\mathrm{p}=0.001)$. We then measured WHR, which in group I, II and III was $0.753 \pm 0.09,0.97 \pm 0.088$ and $1.177 \pm$ 0.11 , respectively $(\mathrm{p}=0.01)$. Compared with DMT2 and controls, there was a significant difference in fasting blood glucose levels, SBP and DBP in DMT2HTN patients ( $p<0.001)$.

Table 1. Clinical and laboratory data among healthy controls and patients with type 2 diabetics without hypertension and type

\begin{tabular}{|c|c|c|c|c|}
\hline Parameter & $\begin{array}{l}\text { Healthy Controls } \\
\qquad(\mathbf{n}=35)\end{array}$ & $\begin{array}{c}\text { Type } 2 \text { diabetics } \\
\text { without hypertension, } \\
\text { (DMT2) } \\
(n=35)\end{array}$ & $\begin{array}{c}\text { Type } 2 \text { diabetics with } \\
\text { hypertension, } \\
\text { (DMT2HTN) } \\
(n=35)\end{array}$ & $p$ value \\
\hline Age (years) & $49.285 \pm 8.043$ & $53.657 \pm 7.534$ & $54.742 \pm 5.793$ & 0.06 \\
\hline Body mass index (Kg/m2) & $19.238 \pm 3.231$ & $24.68 \pm 2.515$ & $31.043 \pm 3.641$ & $<0.001$ \\
\hline Waist-hip ratio & $0.753 \pm 0.090$ & $0.970 \pm 0.088$ & $1.177 \pm 0.108$ & $<0.01$ \\
\hline Fasting blood sugar (mg/dl) & $98.8 \pm 9.237$ & $181 \pm 30.675$ & $289.3 \pm 51.998$ & $<0.001$ \\
\hline Systolic blood pressure (mmHg) & $119.486 \pm 18.543$ & $125.143 \pm 8.434$ & $180.514 \pm 8.020$ & $<0.001$ \\
\hline Diastolic blood pressure (mmHg) & $76.343 \pm 5.213$ & $82.4 \pm 5.242$ & $94.971 \pm 5.644$ & $<0.001$ \\
\hline $\begin{array}{l}\text { High-sensitivity C-reactive protein } \\
(\mathrm{mg} / \mathrm{dl})\end{array}$ & $0.299 \pm 0.208$ & $0.844 \pm 0.220$ & $3.507 \pm 0.932$ & $<0.001$ \\
\hline Ischemia modified albumin (IMA) & $0.229 \pm 0.035$ & $0.480 \pm 0,69$ & $0.591 \pm 0.51$ & $<0.001$ \\
\hline Fibrinogen (FIB) (mg/dl) & $234.457 \pm 33.003$ & $488.37 \pm 65.827$ & $651.743 \pm 40.293$ & 0.039 \\
\hline Total cholesterol (mg/dl) & $169.171 \pm 20.008$ & $270.17 \pm 28.474$ & $282.343 \pm 19.085$ & 0.001 \\
\hline Triglycerides (mg/dl) & $124.314 \pm 32.119$ & $180 \pm 12.982$ & $199.457 \pm 19.153$ & 0.001 \\
\hline High density lipoproteins (mg/dl) & $51.514 \pm 11.781$ & $28.571 \pm 7.871$ & $26.543 \pm 5.878$ & $<0.001$ \\
\hline Low density lipoproteins (mg/dl) & $92.25 \pm 26.034$ & $195.314 \pm 39.837$ & $226.657 \pm 33.12$ & $<0.001$ \\
\hline Glycated hemoglobin (mg/dl) & $5.169 \pm 0.590$ & $7.503 \pm 0.904$ & $10.12 \pm 1.540$ & $<0.001$ \\
\hline
\end{tabular}


Additionally, we observed a significant increase in serum hs-CRP in DMT2 patients $(0.844 \pm$ $0.220)$ and DMT2HTN patients $(3.507 \pm 0.932)$ relative to controls $(0.299 \pm 0.208)(\mathrm{p}<0.001)$.

The serum levels of IMA $(\mathrm{p}<0.001)$, FIB $(\mathrm{p}=$ 0.039), TC ( $\mathrm{p}<0.001), \mathrm{TG}(\mathrm{p}=0.001), \mathrm{HDL}(\mathrm{p}<$ $0.001)$, LDL ( $p<0.001)$ and glycated hemoglobin $(p<0.001)$ in DMT2 and DMT2HTN patients increased significantly as well.

The correlation analysis using Karl Pearson's coefficient showed a strong positive correlation of serum hs-CRP to serum cholesterol, TG, LDL, glycated hemoglobin, FBS, BMI and WHR. Serum hs-CRP, however, demonstrated a strong negative correlation to serum HDL, SBP and DBP (Table 2).

Table 2. The correlation between hs- CRP, IMA and FIB with other biochemical parameters in type 2 diabetics without hypertension and type 2 diabetics with hypertension cases.

\begin{tabular}{|c|c|c|c|c|c|c|c|c|c|c|c|c|}
\hline & \multicolumn{12}{|c|}{ Karl - Pearson correlation } \\
\hline & \multicolumn{4}{|c|}{ High-sensitivity C-reactive protein } & \multicolumn{4}{|c|}{ Ischemia-modified albumin } & \multicolumn{4}{|c|}{ Fibrinogen (FIB) } \\
\hline & \multicolumn{2}{|c|}{$\begin{array}{l}\text { Type } 2 \text { diabetics } \\
\text { without } \\
\text { hypertension, } \\
(\mathrm{DMT} 2) \\
(\mathrm{n}=35) \\
\end{array}$} & \multicolumn{2}{|c|}{$\begin{array}{c}\text { Type } 2 \text { diabetics } \\
\text { with hypertension, } \\
(\mathrm{DMT} 2 \mathrm{HTN}) \\
(\mathrm{n}=35)\end{array}$} & \multicolumn{2}{|c|}{$\begin{array}{l}\text { Type } 2 \text { diabetics } \\
\text { without } \\
\text { hypertension, } \\
\text { (DMT2) } \\
(\mathrm{n}=35) \\
\end{array}$} & \multicolumn{2}{|c|}{$\begin{array}{c}\text { Type } 2 \text { diabetics } \\
\text { with hypertension, } \\
(\text { DMT2HTN) } \\
(n=35)\end{array}$} & \multicolumn{2}{|c|}{$\begin{array}{l}\text { Type } 2 \text { diabetics } \\
\text { without } \\
\text { hypertension, } \\
(\mathrm{DMT} 2) \\
(\mathrm{n}=35) \\
\end{array}$} & \multicolumn{2}{|c|}{$\begin{array}{c}\text { Type } 2 \text { diabetics } \\
\text { with hypertension, } \\
(\mathrm{DMT} 2 \mathrm{HTN}) \\
(\mathrm{n}=35)\end{array}$} \\
\hline & $\mathbf{r}$ & $\mathbf{p}$ & $\mathbf{r}$ & $\mathbf{p}$ & $\mathbf{r}$ & $\mathbf{p}$ & $\mathbf{r}$ & $\mathbf{p}$ & $\mathbf{r}$ & $\mathbf{p}$ & $\mathbf{r}$ & $\mathbf{p}$ \\
\hline $\mathrm{TC}$ & 0.457 & 0.005 & 0.788 & $<0.001$ & 0.658 & $<0.001$ & 0.967 & $<0.001$ & 0.379 & 0.005 & 0.389 & $<0.001$ \\
\hline TG & 0.958 & $<0.001$ & 0.898 & $<0.001$ & 0.715 & $<0.001$ & 0.989 & $<0.001$ & 0.57 & $<0.001$ & 0.584 & $<0.001$ \\
\hline HDL & -0.874 & $<0.001$ & -0.133 & 0.445 & -0.837 & $<0.001$ & -0.107 & 0.047 & -0.654 & $<0.001$ & -0.611 & 0.033 \\
\hline LDL & 0.918 & $<0.001$ & 0.707 & $<0.001$ & 0.768 & $<0.001$ & 0.902 & $<0.001$ & 0.658 & $<0.001$ & 0.628 & $<0.001$ \\
\hline $\mathrm{HbA1C}$ & 0.937 & $<0.001$ & 0.742 & $<0.001$ & 0.661 & $<0.001$ & 0.912 & $<0.001$ & 0.588 & $<0.001$ & 0.548 & $<0.001$ \\
\hline FBS & 0.645 & $<0.001$ & 0.438 & 0.008 & 0.612 & $<0.001$ & 0.982 & $<0.001$ & 0.449 & 0.005 & 0.491 & $<0.001$ \\
\hline SBP & -0.0209 & 0.227 & 0.877 & $<0.001$ & -0.0443 & 0.008 & -0.453 & 0.007 & -0.18 & 0.303 & -0.18 & 0.004 \\
\hline DBP & 0.145 & 0.407 & 0.568 & $<0.001$ & 0.124 & 0.476 & 0.304 & 0.081 & -0.04 & 0.817 & 0.322 & 0.063 \\
\hline BMI & 0.625 & $<0.001$ & 0.204 & 0.24 & 0.44 & 0.008 & 0.966 & $<0.001$ & 0.488 & 0.003 & 0.488 & $<0.001$ \\
\hline WHR & 0.85 & $<0.001$ & 0.496 & 0.002 & 0.794 & $<0.001$ & 0.99 & $<0.001$ & 0.599 & $<0.001$ & 0.579 & $<0.001$ \\
\hline
\end{tabular}

TC, Total cholesterol; TG, Triglycerides; HDL, High-density lipoproteins; LDL, Low-density lipoproteins; HbAlC, Glycated hemoglobin; FBS, Fasting Blood Sugar; SBP, Systolic blood pressure; DBP, Diastolic blood pressure; BMI, Body mass index; WHR, Waist-hip ratio.

Serum IMA showed a strong association to WHR and serum cholesterol, TG and LDL, HDL, FBS, glycated hemoglobin levels. There was, however, a negative correlation between SBP, DBP and BMI to serum IMA. While serum IMA showed a positive correlation to DBP, the correlation was not significant.

\section{Discussion}

Throughout the study, levels of serum hs-CRP, IMA and FIB levels were estimated in DMT2 and DMT2HTN patients and compared to healthy individuals. Levels were then correlated to certain biochemical parameters including TC, TG, HDL, LDL, HbA1C, glycated hemoglobin, and FBS and physical parameters such as SBP, DBP, BMI and WHR. These factors were eventually assessed for possible association with DMT2 and DMT2HTN patients.

Elevated BP is a major complication in patients with diabetes and the prevalence may vary depending on demographics, type and length of diabetes. Risk factors related to HT may lead to the development of severe heart failure, cardiovascular and microvascular complications (10). Since HT is commonly seen in diabetes patients $(4,11)$, early intervention may be appropriate and the existence of different risk factors and the patient's particular lab parameter profile should be considered. 
For DMT2 and DMT2HTN patients, relative to controls, serum hs-CRP levels were significantly increased. CRP is a liver-producing pentameric protein that is considered the 'golden marker for inflammation.' Several studies indicate a possible link between HT, diabetes and inflammation (1113). CRP reacts with the capsule polysaccharide of Streptococcus pneumoniae (14), is an acute phase response protein, that becomes markedly increased during inflammatory and infectious diseases (15), and key player of innate immunity, for example, CRP is involved in complement binding, but also induces phagocytosis, and apoptosis (16). The hsCRP levels in DMT2HTN individuals were higher than in normal subjects, indicating that DMT2HTN patients had a hyperactive inflammatory condition (17).

IMA is a novel marker of ischemic injury and oxidative damage. Serum IMA levels were increased significantly in DMT2 and DMT2HTN individuals compared with controls. In addition, these findings were similar to those of Piwowar A et al (18). Moreover, Shao-gang et al., showed a significant elevation and positive correlation of IMA in peripheral arterial disease patients with T2DM (19). In DMT2 patients, Kaefer et al., found that elevated IMA was significantly corrected with blood glucose, glycated albumin and CRP (6). Interestingly, IMA can detect hyperglycemiainduced oxidative stress before the clinical symptoms of diabetes related complications become evident. Low-grade chronic inflammation and endothelial dysfunction are contributing factors to cardiovascular disease initiation and progression. Here, IMA was measured based on cobalt binding of serum albumin. IMA is not tissue specific and is elevated in subjects with oxidative stress, rather than cardiac ischemia (20).

There were concerns about the tissue-specificity of IMA when it was suggested that IMA is a biomarker for other diseases related to oxidative stress or ischemia. In previous studies, the possible role of IMA has been confirmed (21). We observed that T2DM patients had impaired glycemic control, which was correlated with increased biomarkers of inflammatory and oxidative stress. Firstly, higher levels of IMA and CRP were found in T2DM patients. Hyperglycemia and inflammation may have decreased albumin's ability to bind cobalt, leading to higher serum levels of IMA (6). Based on the above data, IMA could be an important biomarker for oxidative stress in T2DM and T2DMHTN patients. Furthermore, increased serum IMA was found in hyperglycemic patients. Further, there was a correlation between IMA and CRP (22). CRP is regulated by pro-inflammatory cytokines including interleukin-6 (IL-6), IL-1 $\beta$, and tumor necrosis factor alpha (TNF- $\alpha)$. The mechanisms of CRP and IMA production differ in certain aspects. Here, in patients without HT, we found that IMA showed a strong positive correlation to TC, TG, LDL, FBS, and hbA1c, but a negative correlation to SBP, DBP and BMI. Therefore, the sensitivity and specificity of IMA depends on the patient's unique clinical features. The contributing factors are being investigated for this observation, but further studies are needed to investigate the exact role of IMA in T2DM patients because for this specific sub-set, it still remains unclear.

Next, we observed a significant increase in serum FIB levels in T2DM and T2DMHTN patients, relative to controls. These findings coincided with the results by Ganda op et al (23). Serum FIB showed a strong association between TC, TG, LDL, FBS and HbA1c, but a negative correlation to HDL. The intermolecular crosslinking of collagen in FIB is a major complication of chronic diabetes, as it leads to the stiffening of cardiovascular tissue. Macrovascular and microvascular disease and glycemic control are associated with factors such as the plasminogen activator inhibitor 1, von-Willebrand, FIB, factor VII and thrombin-antithrombin complexes (24).

In diabetics, plasma levels of lipoprotein A are elevated, particularly those with poor glycemic control. The lipoprotein A molecule is formed by the assembly of at least two major proteins, specifically, a molecule of apoB100 covalently connected by a single disulfide bridge with an apolipoprotein A molecule. It is structurally similar to LDL in protein and lipid composition, with apolipoprotein as the only difference. Apolipoprotein, a glycoprotein structurally similar to plasminogen and the precursor of plasmin, has the capacity to bind fibrin and membrane proteins of endothelial cells and monocytes. This inhibits plasminogen binding and plasmin generation, 
leading to decreased fibrinolysis, delayed thrombolysis and the accumulation of lipoprotein A and fibrin at sites of vascular injury. By decreasing fibrinolysis and therefore increasing plasma FIB levels, lipoprotein A plays major role in diabetes and associated vascular complications. The mechanism by which FIB increases cardiovascular risks is not fully understood. FIB also plays an important role in atherosclerosis development starting from plaque formation to the formation of occlusive thrombi over a ruptured atherosclerotic plaque, which is the most common cause of myocardial infarction.

T2DM is associated with dyslipidemia. Our analyses found that in DMT2 and DMT2HTN patients, the mean serum levels of TC, TG and LDL were significantly increased. This result is similar to the study by Suryawanshi NP et al (25), which also found a substantial increase in TC, TG and LDL.

The rise in TC could be attributed to a reduction in muscle activity and suppression of cholesterol catabolism. Increased TG content may have occurred due to increased mobilization of fatty acids for energy from adipose tissue to the liver, where excess fatty acids are converted to TG.

Insulin increases the number of LDL receptors, and chronic insulin deficiency may be associated with a decreased level of LDL receptors, causing LDL-cholesterol levels to rise. Serum HDL cholesterol levels in DMT2 and DMT2HTN groups were found to be significantly lower compared to controls. These findings were consistent with Aabdel-Aal NM (26). Insulin resistance contributes to increased HDL catabolism in the presence of normal amounts of cholesterol ester transport protein (CETP) and hepatic lipase. The decreased levels may also be due to decreased production, secondary to impaired catabolism of very-low-density lipoprotein (VLDL) and decreased lipoprotein lipase activity.

When plasma glucose is consistently elevated, there is an increase in nonenzymatic glycation of hemoglobin. HbA1C is formed by an irreversible nonenzymatic mechanism in which an aldehyde group of a glucose molecule bonds with a terminal valine residue on the $\beta$ chain of hemoglobin. The present study showed a substantial rise in serum glycated hemoglobin levels in DMT2 and
DMT2HTN groups compared to controls. These observations also coincided with the results described by Shetty JK et al (27).

Our analysis found that when compared to controls, BMI and WHR were significantly increased in DMT2 and DMT2HTN groups. Similar results were obtained in several studies (2830) suggesting that BMI and WHR may be early predictors of diabetes and HT. BMI, WHR, and hsCRP levels in both DMT2 and DMT2HTN groups showed a strong positive correlation with serum TC, TG and LDL. Further, hs-CRP showed a strong negative correlation to serum HDL. One risk factor for cardiovascular disease is dyslipidemia (31). Such patients may also have underlying cardiovascular complications and may explain the correlation of hs-CRP to the observed lipid profile in this study.

The IMA levels in both DMT2 and DMT2HTN groups showed a strong positive correlation with FBS, glycated hemoglobin, BMI and WHR. These findings were similar to the findings outlined in Kaefer $\mathrm{M}$ et al (6). In T2DM individuals, serum IMA levels were increased which is independent of other clinical variables and may lead to the accelerated progression of cardiovascular events. In DMT2 and DMT2HTN patients, IMA levels showed a strong positive correlation to serum TC, TG and LDL but a strong negative correlation to serum HDL, similar to Dahiya et al (32). In DMT2 patients, there was a strong correlation between IMA and study parameters such as serum TC, TG, HDL, LDL and WHR. There was a correlation of IMA and BMI in DMT2HTN individuals, however, it was not significant. Lastly, serum IMA showed a strong negative correlation to SBP and DBP.

Similar to Burno $\mathrm{G}$ et al., we found that FIB levels in both DMT2 and DMT2HTN groups showed a strong positive correlation to TC, TG, LDL, FBS, glycated hemoglobin, BMI and WHR (33). Patients with T2DM and HT are often subject to chronic oxidative stress. Lipid peroxidation of cellular structures is an essential process in atherosclerosis and may occur as a late complication of diabetes mellitus (DM). FIB, a major factor in LDL modification, is the byproduct of arachidonic, eicosapentaenoic and docosahexaenoic acid peroxidation. LDL oxidation 
occurs in apoB-100 (LDL) via the interactions between aldehyde and lysine residues (34). The pathological effects of oxidized LDL include the induction of atherosclerosis (by stimulating monocyte infiltration and smooth muscle cell migration and proliferation), atherothrombosis (by inducing apoptosis of endothelial cell), and plaque erosion (by impairing the endothelial anticoagulant balance) (35).

Finally, in both DMT2 and DMT2HTN individuals, we observed a strong positive correlation between serum FIB levels and serum TC, serum TG and serum LDL. Similar to Ganda et al, FIB also demonstrated a strong negative correlation to serum HDL (23). In diabetic subjects with vascular complications, a greater level of lipid peroxides was observed. This increase in lipid peroxide may be due to the increased free radical formation activity. Free radical interactions results in the development of toxic endoperoxides during arachidonic acid metabolism (36). The formed lipid peroxide stimulates the synthesis of cyclooxygenase, prostaglandins and thromboxane. This will cause increased accumulation of platelets and lead to complications in the vascular system. Furthermore, lipid peroxide may increase proportionally to blood glucose levels. The increase in lipid peroxidation and prolonged hyperglycemic conditions may be explained by the presence of superoxide dismutase enzymes, antioxidants which become inactive due to the

\section{References}

1. Unnikrishnan R, Pradeepa R, Joshi SR, Mohan V. Type 2 Diabetes: Demystifying the Global Epidemic. Diabetes. 2017;66(6):1432-1442.

2. Basu S, Yudkin JS, Kehlenbrink S, Davies JI, Wild SH, Lipska KJ, et al. Estimation of global insulin use for type 2 diabetes, 2018-30: a microsimulation analysis. The lancet Diabetes \& endocrinology. 2019;7(1):25-33.

3. Wu Y, Ding Y, Tanaka Y, Zhang W. Risk factors contributing to type 2 diabetes and recent advances in the treatment and prevention. International journal of medical sciences. 2014;11(11):1185-1200.

4. Ohishi M. Hypertension with diabetes mellitus: physiology and pathology. Hypertension research. formation of superoxide radicals within the cell (37). Maximum lipid peroxidation contributes to tissue and organ damage, a common complication in diabetics. TC may appear high due to increased cholesterol synthesis, and changes in TG levels may be correlated to glycemic control.

As more than $80 \%$ of T2DM patients develop HT, and about $20 \%$ of HT patients develop diabetes, a significant proportion of cardiovascular related morbidity and mortality cases could be accounted for by a combination of cardiovascular risk factors (38). Therefore, the use of one or more biomarker such as IMA, FIB and hs-CRP could be used during routine cardiovascular risk assessments as diagnostic biomarkers.

Future clinical studies are required to show whether outcomes can be improved when serum IMA, FIB, and hs-CRP are used together for prognostic evaluation and therapeutic decisionmaking.

Overall, the study indicates an irregular lipid profile with hypercholesterolemia, hypertriglyceridemia, elevated LDL, low HDL, and impaired glycemic control in type 2 diabetes mellitus patients with and without HT.

\section{Acknowledgment}

Authors are thankful to all the patients and volunteers who participated in this study.

The authors declare that they have no conflict of interest.

2018;41(6):389-393.

5. Khangura DS, Waqar Salam M, Brietzke SA, Sowers JR. Hypertension in Diabetes. In: Feingold KR, Anawalt B, Boyce A, Chrousos G, Dungan K, Grossman A, et al., editors. Endotext. South Dartmouth (MA)2000.

6. Kaefer M, Piva SJ, De Carvalho JA, Da Silva DB, Becker AM, Coelho AC, et al. Association between ischemia modified albumin, inflammation and hyperglycemia in type 2 diabetes mellitus. Clinical biochemistry. 2010;43(4-5):450-4.

7. Shah S, Kalal BS. Oxidative stress in cervical cancer and its response to chemoradiation. Turkish journal of obstetrics and gynecology. 2019;16(2):124-8. 
8. Bar-Or D, Lau E, Winkler JV. A novel assay for cobalt-albumin binding and its potential as a marker for myocardial ischemia-a preliminary report. $\mathrm{J}$ Emerg Med. 2000;19(4):311-5.

9. Vaishya R, Arora S, Singh B, Mallika V. Modification of Jaffe's kinetic method decreases bilirubin interference: A preliminary report. Indian J Clin Biochem. 2010;25(1):64-66.

10. de Boer IH, Bangalore S, Benetos A, Davis AM, Michos ED, Muntner P, et al. Diabetes and Hypertension: A Position Statement by the American Diabetes Association. Diabetes care. 2017;40(9):1273-1284.

11. Lima LM, Carvalho M, Soares AL, Sabino Ade P, Fernandes AP, Novelli BA, et al. High-sensitivity C-reactive protein in subjects with type 2 diabetes mellitus and/or high blood pressure. Arquivos brasileiros de endocrinologia \& metabologia. 2007;51(6):956-60.

12. Ebong IA, Schreiner P, Lewis CE, Appiah D, Ghelani A, Wellons M. The association between high-sensitivity C-reactive protein and hypertension in women of the CARDIA study. Menopause. 2016;23(6):662-668.

13. Tutuncu Y, Satman I, Celik S, Dinccag N, Karsidag K, Telci A, et al. A Comparison of hsCRP Levels in New Diabetes Groups Diagnosed Based on FPG, 2-hPG, or HbAlc Criteria. J Diabetes Res. 2016;2016:5827041.

14. Nagaraj S, Kalal BS, Manoharan A, Shet A. Streptococcus pneumoniae serotype prevalence and antibiotic resistance among young children with invasive pneumococcal disease: experience from a tertiary care center in South India. Germs. 2017;7(2):78-85.

15. Nishana E, Bhat SS, Sahana KS, Hegde SK, Bhat V, Kalal BS. Estimation of Salivary SCD14 in Children with Early Childhood Caries in Association with Pneumonia. Rep Biochem Mol Biol. 2019;8(2):132-138.

16. Sproston NR, Ashworth JJ. Role of C-Reactive Protein at Sites of Inflammation and Infection. Front Immunol. 2018;9:754.

17. Oh J, Teoh H, Leiter LA. Should C-reactive protein be a target of therapy? Diabetes care. 2011;34(Suppl 2):S155-S160.
18. Piwowar A, Knapik-Kordecka M, Warwas M. Ischemia-modified albumin level in type 2 diabetes mellitus- Preliminary report. Disease markers. 2008;24(6):311-317.

19. Ma SG, Wei CL, Hong B, Yu WN. Ischemiamodified albumin in type 2 diabetic patients with and without peripheral arterial disease. Clinics (Sao Paulo). 2011;66(10):1677-1680.

20. Ukinc K, Eminagaoglu S, Ersoz HO, Erem C, Karahan C, Hacihasanoglu AB, et al. A novel indicator of widespread endothelial damage and ischemia in diabetic patients: ischemia-modified albumin. Endocrine. 2009;36(3):425-32.

21. Rodrigo R, Libuy M, Feliu F, Hasson D. Oxidative stress-related biomarkers in essential hypertension and ischemia-reperfusion myocardial damage. Dis markers. 2013;35(6):773-790.

22. Jia ZT, Liu CY, Li H. Changes of the concentration of serum ischemia modified albumin and high sensitivity C-reactive protein in type 2 diabetic patients with retinopathy. Zhonghua yan ke za zhi. 2009;45(9):805-8.

23. Ganda OP, Arkin CF. Hyperfibrinogenemia. An important risk factor for vascular complications in diabetes. Diabetes care. 1992;15(10):1245-50.

24. Bembde AS. A study of plasma fibrinogen level in type-2 diabetes mellitus and its relation to glycemic control. Indian J Hematol Blood Transfus. 2012;28(2):105-108.

25. Suryawanshi NP, Bhutey AK, Nagdeote AN, Jadhav AA, Manoorkar GS. Study of lipid peroxide and lipid profile in diabetes mellitus. Indian J Clin Biochem. 2006;21(1):126-130.

26. Abdel-Aal NM, Ahmad AT, Froelicher ES, Batieha AM, Hamza MM, Ajlouni KM. Prevalence of dyslipidemia in patients with type 2 diabetes in Jordan. Saudi Med J. 2008;29(10):1423-8.

27. Shetty JK, Prakash M, Ibrahim MS. Relationship between free iron and glycated hemoglobin in uncontrolled type 2 diabetes patients associated with complications. Indian $\mathbf{J}$ Clin Biochem. 2008;23(1):67-70.

28. Radzeviciene L, Ostrauskas R. Body mass index, waist circumference, waist-hip ratio, waist-height ratio and risk for type 2 diabetes in women: a casecontrol study. Public health. 2013;127(3):241-6. 
29. Sigdel M, Kumar A, Gyawali P, Shrestha R, Tuladhar ET, Jha B. Association of high sensitivity C-reactive protein with the components of metabolic syndrome in diabetic and non-diabetic individuals. J Clin Diagn Res. 2014;8(6):CC11-CC13.

30. Peixoto Mdo R, Benicio MH, Latorre Mdo R, Jardim PC. Waist circumference and body mass index as predictors of hypertension. Arq Bras Cardiol. 2006;87(4):462-70.

31. Nepal G, Tuladhar ET, Acharya K, Bhattarai A, Sharma VK, Raut M, et al. Dyslipidemia and Associated Cardiovascular Risk Factors among Young Nepalese University Students. Cureus. 2018;10(1):e2089.

32. Dahiya K, Aggarwal K, Seth S, Singh V, Sharma TK. Type 2 diabetes mellitus without vascular complications and ischemia modified albumin. Clin Lab. 2010;56(5-6):187-90.

33. Bruno G, Cavallo-Perin P, Bargero G, Borra M, D'Errico N, Pagano G. Association of fibrinogen with glycemic control and albumin excretion rate in patients with non-insulin-dependent diabetes mellitus. Ann Intern Med. 1996;125(8):653-7.
34. Linton MRF, Yancey PG, Davies SS, Jerome WG, Linton EF, Song WL, et al. The Role of Lipids and Lipoproteins in Atherosclerosis. In: Feingold KR, Anawalt B, Boyce A, Chrousos G, Dungan K, Grossman A, et al. Endotext. South Dartmouth (MA): MDText.com, Inc.; 2000.

35. Shetty R, Rai M, Chandrashekar R, Kalal BS. Diabetogenic effect of gluten in Wistar albino rats: a preliminary preclinical screening. Med Pharm Rep. 2020;93(1):47-52.

36. Kalal BS, Fathima F, Pai VR, Sanjeev G, Krishna CM, Upadhya D. Inhibition of ERK1/2 or AKT Activity Equally Enhances Radiation Sensitization in B16F10 Cells. World J Oncol. 2018;9(1):21-28.

37. Matough FA, Budin SB, Hamid ZA, Alwahaibi $\mathrm{N}$, Mohamed J. The role of oxidative stress and antioxidants in diabetic complications. Sultan Qaboos Univ Med J. 2012;12(1):5-18.

38. Lastra G, Syed S, Kurukulasuriya LR, Manrique C, Sowers JR. Type 2 diabetes mellitus and hypertension: an update. Endocrinol Metab Clin North Am. 2014;43(1):103-122. 